\title{
Recent Developments in Pharmacotherapy for Vasomotor Symptoms
}

\author{
Wulf H. Utian
}

Published online: 21 February 2012

(C) Springer Science+Business Media, LLC 2012

\begin{abstract}
Many women experience vasomotor symptoms (VMS) at or around the time of menopause. Hot flashes and night sweats are considered primary menopausal symptoms that may also be associated with sleep and mood disturbances, as well as decreased cognitive function. All of these symptoms may lead to social impairment and workrelated difficulties that significantly decrease overall quality of life. Hot flashes have shown a great deal of variability in their frequency and severity in women. In some women, hot flashes persist for several months, but in others, they may last for more than 10 years. Traditionally, VMS were reported to begin after menopause, but night sweats in particular most often begin in perimenopause, several years before the final period. The pathogenesis of hot flashes has not yet been fully elucidated. Hormonal therapy for menopauseassociated VMS has been the mainstay for the management of these symptoms for more than 50 years. However, because many women now want to avoid hormone therapy, there is a need for additional targeted therapies, validated by results from controlled clinical trials, that are safe, efficacious, cost-effective, and well tolerated by symptomatic menopausal women. The current status of these new pharmacotherapies for VMS is reviewed.
\end{abstract}

Keywords Vasomotor symptoms · Menopause $\cdot$ Hot flashes $\cdot$ Hot flushes $\cdot$ Night sweats $\cdot$ Estrogen $\cdot$ Hormone therapy $\cdot$ Isoflavones $\cdot \beta$ receptor agonist $\cdot$ Risk factors . Treatment $\cdot$ Pharmacotherapy $\cdot$ CAM $\cdot$ TSEC $\cdot$ Gabapentin . Eszopiclone $\cdot$ MF-101 $\cdot$ Equol

\footnotetext{
W. H. Utian $(\bowtie)$

Point East P7 27500 Cedar Road,

Beachwood, OH 44122, USA

e-mail: wulf@utianllc.com
}

\section{Introduction}

Menopause is characterized by physiologic and psychosocial changes in a woman's life. Menopause may be associated with vasomotor symptoms (VMS), which include hot flashes (also referred to as hot flushes) and night sweats, as well as bone loss, urogenital atrophy, urinary tract infections and incontinence, increased cardiovascular risk, somatic symptoms, sexual dysfunction and decreased libido, and loss of skin elasticity. VMS, and the sleep and mood disturbances that often result from them, can have a significant negative impact on overall quality of life (QOL) for a substantial number of women. The impact of VMS has gained in importance as the lifespan of women has increased throughout the world, as women can expect to spend a significant portion of their lives after menopause. This period should be a highly productive time for women, and maintaining functional ability and a good QOL is of utmost importance. Accordingly, it is important that safe, efficacious, cost-effective, and well-tolerated treatments are made available. Thus, this paper briefly reviews the epidemiology of VMS and what is known about the physiologic basis of these symptoms, as background to description of the current status of recent drugs for VMS that are newly available or under development, including complementary and alternative products.

\section{Epidemiology of Vasomotor Symptoms}

Prevalence and Risk Factors

US Census Bureau statistics indicate that approximately one third of women are older than 50 years of age [1]. It is estimated that $75 \%$ of women in this age group will 
experience hot flashes, a value supported by a recent longitudinal study of 454 women who were followed from premenopause to postmenopause [2]. Worldwide, between $50 \%$ and $85 \%$ of women (approximately 360 million) older than 45 years of age experience hot flashes [3]. The prevalence of hot flashes varies widely across populations and is strongly influenced by culture and ethnicity. In the United States, the Study of Women's Health Across the Nation (SWAN) surveyed more than 16,000 women and found that the prevalence of hot flashes was highest among African Americans (46\%), followed by Hispanics (34\%), whites (31\%), Chinese (21\%), and Japanese (18\%) [4]. In other parts of the world, rates of hot flashes vary widely as well, with the lowest prevalence observed in China $(10 \%)$ and other Asian nations [5].

Many attempts have been made to identify demographic characteristics associated with a significantly increased risk of hot flashes. For many years, low body mass index (BMI) and race were considered significant predictors of VMS, with thin, white women believed to at the highest risk for hot flashes. More recent findings have suggested that high BMI and African American race are associated with a higher risk of VMS. This shift may be related to better sampling of the general population by major clinical trials because white middle-class women traditionally participated in clinical trials that often did not include women from other ethnic groups. The multiethnic SWAN not only demonstrated a link between an elevated BMI $\left(\geq 27 \mathrm{~kg} / \mathrm{m}^{2}\right)$ and hot flashes [5] but also showed an increased prevalence in African American women, as mentioned. Ongoing studies continue to investigate potential predictors of hot flashes. Smoking, maternal history, history of premenstrual complaints, elevated basal core body temperature, low physical activity, low socioeconomic status, and low levels of estrogen and high levels of luteinizing and follicle-stimulating hormones prior to the menopausal transition have all been associated with an increased risk of hot flashes [4, 6-8].

\section{Timing of Hot Flashes}

Several researchers have reviewed the timing and frequency of hot flashes [9]. SWAN demonstrated that hot flashes occur earlier than previously believed and may become less frequent and less intense as menopause progresses. SWAN data indicated that VMS were more frequently reported by women in late perimenopause, with a relative risk for hot flashes at 1.0 during premenopause (the 1 or 2 years prior to menopause), 2.06 during early perimenopause (the early menopausal transition), 4.32 during late perimenopause (the late menopausal transition), and 2.81 during postmenopause [4]. The frequency of hot flashes varies but tends to remain consistent for an individual. Many women have hot flashes on a daily basis, some as frequently as every hour, whereas others have VMS infrequently (i.e., weekly or monthly) [10]. The majority of women report experiencing hot flashes for 6 months to 2 years, with the highest number of women reporting symptoms during the first 2 postmenopausal years. In another study, however, $26 \%$ of women reported having hot flashes for 6 to 10 years and $10 \%$ reported having had VMS for more than 10 years [11].

\section{Pathophysiology}

The cause of hot flashes has yet to be determined because of the limited research focus in this therapeutic area. Hot flashes are believed to result from the brain's response to diminished hormones and hormonal fluctuations that occur during the menopausal transition. Ovarian hormones have been shown to influence thermoregulatory mechanisms that regulate temperature homeostasis in the hypothalamus. The neurotransmitters serotonin and norepinephrine play a role in modulating core body temperature, neurochemical messaging, and peripheral vasculature [12]. Several investigators have documented cardiovascular, temperature, hormonal, and autonomic parameters with hot flashes and link them with thermoregulatory mechanisms [12-14].

\section{Quantification of VMS and Their Impact on Quality of Life}

Perceived QOL is difficult to measure, and there is no universal agreement on how it should be quantified. Objective measurements of health status (often referred to as Health-Related QOL [HRQOL]) may not capture the patient's perception of overall life satisfaction. HRQOL may be viewed as the individual's perception regarding her physical, cognitive, and mental health as well as her social situation [15]. Consideration of HRQOL is also influenced by women's increased risk of multiple chronic diseases associated with menopause, including osteopenia, osteoporosis and related fractures, and cardiovascular disease [16]. Assessments of overall QOL for menopausal women must consider not only somatic symptoms (hot flashes, night sweats, urogenital atrophy), but also psychological symptoms (depression, mood swings, irritability, anxiety), and life circumstances (function in the workplace), to give a full measure of QOL, or Global QOL.

VMS can have a significant negative impact on QOL in younger and older women, contributing to physical and psychosocial impairment. Becoming flushed and sweating profusely in a social or work-related situation may cause extreme anxiety for many women and lead to social isolation [17]. Unpredictable hot flashes may result in increased anxiety and stress through sleep deprivation and mood 
swings. A large number of studies have documented the negative impact of menopause on QOL $[18,19]$.

This aspect is emphasized because of the high level of placebo response in randomized, double-blind, placebocontrolled drug studies. This response can be a cause of confusion in interpreting outcomes. For example, the active arm in a trial may appear to have significant efficacy if the comparative placebo arm has a low level of response, but the same drug may appear to be ineffective if the level of response in the placebo arm is high.

\section{Impact of VMS on Sleep, Mood, and Cognitive Function}

Despite the lack of agreement in the medical literature about the relationship between VMS and sleep quality, mood variability, and cognitive function, these symptoms are primary complaints that menopausal women present to their healthcare practitioners. The causes of menopause-related sleep disturbances are controversial. Sleep disturbances have been related to hormonal changes that trigger hot flashes or night sweats, independent of age [20-22].

\section{Women's Approach to Treatment}

Before seeking medical advice at the onset of VMS, women are likely to obtain information from their peers, family members, or the Internet, and their attitudes may be influenced by the premature terminations of the hormone arms of the Women's Health Initiative (WHI) [23]. Many women resort to self-diagnosis and treatment, combining over-thecounter drugs with medications prescribed for other conditions (e.g., analgesics for headache; anxiolytics and antidepressants for anxiety, tension, and mood changes; sedatives/ hypnotics for insomnia). Most of these treatments fail to provide significant relief of VMS, and many of these women ultimately consult their physicians after these remedies are unsuccessful.

\section{Advances in Pharmacotherapy}

\section{Hormonal Therapy}

Estrogen therapy (ET) and estrogen-progestogen therapy (EPT) remain the gold standard for treatment of VMS, with an average $95 \%$ reduction in frequency and number of VMS. For women within 5 years of menopause, ET and EPT are also quite safe if the guidelines of risks and benefits presented by The North American Menopause Society (NAMS) are followed [24••]. Considerable research over many years has clearly defined and answered most of the key questions around ET/EPT, and the barrier to new therapies having equal efficacy and long-term data is therefore very high $[25 \cdot \bullet]$. New developments in the area of estrogen and estrogen-progestogen combinations have essentially been limited to routes of delivery and/or lower doses.

\section{Estrogen Agonists/Antagonists}

With the approval of indications for prevention of breast cancer for tamoxifen and raloxifene (as well as osteoporosis prevention for the latter), it was hoped that estrogen agonists/antagonists (formerly called selective estrogen receptor modulators, SERMs) could be developed that would have the breast, endometrium, and bone benefits and would also alleviate VMS. Unfortunately, the molecules studied thus far have not shown adequate efficacy against VMS $[26,27]$.

\section{Tissue-Selective Estrogen Complex}

A tissue-selective estrogen complex (TSEC) partners bazedoxifene (BZA), a novel estrogen agonist/antagonist, with conjugated estrogens (CE), with the goal of designing a novel menopausal therapy that combines the beneficial effects of both [28]. Ideally, a TSEC would relieve menopausal symptoms such as hot flushes and vaginal atrophy, prevent osteoporosis, and have a favorable safety and tolerability profile, including a neutral effect on the breast and uterus. BZA is a novel estrogen agonist/antagonist that has shown efficacy in preventing fractures and preserving bone mineral density (BMD) in postmenopausal women, without any evidence of endometrial or breast stimulation [28], $[29 \bullet \cdot, 30,31 \bullet \cdot$. The efficacy and safety of BZA/CE have been assessed in a series of phase 3 trials. In postmenopausal women with a uterus, varying doses of BZA/CE were shown to increase BMD and improve measures of vulvar/ vaginal atrophy and sexual function without evidence of endometrial stimulation or an increase in uterine bleeding. A recent study of symptomatic postmenopausal women showed that BZA $20 \mathrm{mg}$ with CE 0.45 or $0.625 \mathrm{mg}$ significantly reduced the daily number of moderate-to-severe hot flushes compared with placebo after 12 weeks $(74 \%$ and $80 \%$ from baseline, respectively; $P<0.001$ vs placebo). In addition, both doses of BZA/CE significantly decreased the severity of hot flushes relative to placebo as early as Week 3 ; this reduction was sustained through 12 weeks [29••].

\section{Other Prescription Medications}

Other prescription medications approved for use in conditions not associated with menopause-related VMS have demonstrated varying degrees of efficacy [32]. The economic advantage for some of these medications is that they have 
been on the market for a number of years. Given the understanding that VMS are the result of a dysfunction in thermoregulatory circuitry, new nonhormonal therapies that selectively target the serotonin and norepinephrine pathways, without the involvement of other pathways, have been the subject of some scrutiny.

\section{SSRIs and SNRIs}

Selective serotonin reuptake inhibitors (SSRIs) and serotonin-norepinephrine reuptake inhibitors (SNRIs) have received increased attention for the management of VMS in nondepressed menopausal women. Initial studies were largely undertaken in breast cancer survivors, and the number and severity of VMS were less than the standards required by the US Food and Drug Administration (FDA) and utilized in randomized controlled studies of hormone therapies [32-36]. Efficacy in reducing the number and severity of VMS averages around 65\% with SSRIs like fluoxetine $(20 \mathrm{mg} / \mathrm{d})$ and paroxetine $(12.5-25 \mathrm{mg} / \mathrm{d})$, and an SNRI like venlafaxine (37.5-75 mg/d).

A more recent product, desvenlafaxine, is also under review by the FDA. A significant decrease from baseline in the number of VMS occurred at weeks 4 and 12 with desvenlafaxine compared with placebo (week 12 reductions: $60 \%$ with $100 \mathrm{mg}$ of desvenlafaxine, $66 \%$ with $150 \mathrm{mg}$, and $47 \%$ with placebo; all $P \leq 0.002$ ). Compared with placebotreated women, significantly more women treated with desvenlafaxine discontinued treatment because of adverse events during week 1 only [37••].

A serious limitation on the use of these drugs as a firstline treatment is the absence of any long-term research as to what happens when the drugs are withdrawn in nondepressed women being treated for VMS. For this reason, caution is urged regarding their use. Indeed, sudden withdrawal has been associated with headaches and anxiety, so they should not be stopped abruptly but rather tapered for at least 2 weeks. On the other hand, when VMS and appropriately diagnosed mood disorders such as depression are both present, these drugs may be beneficial.

Limitations on the use of these compounds include loss of libido, drowsiness, weight gain (paroxetine), weight loss (venlafaxine), and nausea. To minimize potential adverse effects, treatment should always be started with the lowest dose, which should be increased only if there has been no response after several weeks. Doses higher than those used in clinical studies are not justified, especially as the risk of toxicity may increase with the dose.

\section{Hypnotics}

Eszopiclone is a hypnotic or sleep aid. Perimenopausal and postmenopausal women with sleep-onset or sleep- maintenance insomnia co-occurring with hot flashes and symptoms of depression or anxiety who were randomized to eszopiclone ( $3 \mathrm{mg}$ orally) or placebo in a double-blinded, 11 -week crossover trial showed improvement $(P<0.05)$ in sleep parameters, depressive symptoms, anxiety symptoms, QOL, and nighttime (but not daytime) hot flashes [38]. Eszopiclone may thus have a role in the treatment of insomnia with co-occurring menopause-related symptoms.

\section{Anticonvulsants}

The anticonvulsant gabapentin has undergone fairly comprehensive study in the treatment of VMS for several years. Most trials were undertaken at lower severity than the FDA usually requires (e.g., at least 14 hot flashes per week rather than the mandated 50 per week). Women receiving 300-mg oral gabapentin capsules or placebo three times daily for 4 weeks, a short testing interval, demonstrated VMS scores that decreased by $51 \%(95 \% \mathrm{CI}, 43 \%-58 \%)$ in the gabapentin group, compared with $26 \%(95 \% \mathrm{CI}, 18 \%-35 \%)$ with placebo, from baseline to week 4 [39]. These women reported greater dizziness (18\%), unsteadiness (14\%), and drowsiness (12\%) at week 1 compared with those taking placebo, but these symptoms improved by week 2 and returned to baseline levels by week 4 [39].

Gabapentin appears to be a weakly effective treatment for hot flashes. Therapy can be initiated at $300 \mathrm{mg} /$ day, with lower doses in women over age 65 . The dose can be gradually increased to up to $300 \mathrm{mg}$ three times per day, for a total dose of $900 \mathrm{mg} /$ day. Because the major adverse effects are drowsiness and dizziness, it would be preferable to begin therapy with dosing at night. As with other psychoactive drugs, tapering is recommended when gabapentin therapy is discontinued.

\section{Complementary and Alternative Medications}

Many symptomatic menopausal women are likely to treat themselves before consulting a medical practitioner, thinking that "natural" products classified as complementary and alternative medications (CAM) are safer and the ingredients more pure than prescription drugs. The most common CAM treatments that have emerged contain individual and compounded formulas of herbs, isoflavones, and dietary supplements that have promised to alleviate menopause-related hot flashes and night sweats, irritability, sleeplessness, mood swings, weight gain, headaches, insomnia, depression, menstrual irregularities, fatigue, and loss of sexual desire. These formulations also claim to promote mental clarity, increase energy levels, and improve physical performance. A key limitation in the analysis of these products is that their clinical efficacy has generally not been documented by results from controlled clinical trials. It has also been noted 
that any benefits associated with herbal supplements may occur more slowly than those achieved with traditional medications [40].

Herbal products that have not withstood scrutiny include dong quai, evening primrose oil, ginseng, and sage. Vitamin $\mathrm{E}$ has also not been effective.

Isoflavonoids

One area of considerable scrutiny has treatment with isoflavonoids derived from soy, flaxseed, or red clover. The conclusion from the recent NAMS/Utian isoflavone translational symposium panel on the role of soy isoflavones in menopausal health was that in postmenopausal women with distressing VMS, initial treatment with isoflavones is reasonable. The starting isoflavone dose should be $50 \mathrm{mg} /$ day or higher, and therapy should be given for at least 12 weeks. Studies of women who do not benefit from soy isoflavones should be undertaken to monitor longer-term beneficial effects or possible adverse effects. If a woman responds to isoflavone supplementation, treatment can continue with monitoring for adverse effects; if a woman does not respond after 12 weeks, other treatment options should be discussed $[41 \cdot \bullet]$.

The most recent randomized, blinded, comparative clinical trial on soy isoflavonoids found them to be no more effective than a placebo. Half of 248 women ages 45 to 60 were given 200 milligrams of soy isoflavones daily and the rest took a placebo. After 2 years, despite urine tests confirming that the women in the soy group had ingested nearly 20 times as much soy as those taking the placebo, there were no significant differences in bone density and no improvement regarding night sweats, insomnia, loss of libido, or vaginal dryness compared with the placebo group. There were few serious adverse effects in either group [42]. This study has been criticized for numerous study-design defects.

It is possible that there is a difference between women who can convert the isoflavone daidzein to equol and hence show efficacy of a supplement versus nonconverting women, who would be unlikely to respond. A deficiency in most studies has been the fact that the study population has not been so defined. A supplement containing natural S-equol may be effective for some women who do not have the capacity to produce equol $[41 \bullet \bullet]$.

\section{In Development: Estrogen Receptor $\beta$ Agonist}

An interesting product in development in the United States is MF-101, a quality-controlled mixture derived from 22 herbs that are traditionally used in Chinese medicine for the treatment of VMS. MF-101 did not promote the growth of breast cancer cells or stimulate uterine cells in preclinical studies [43]. It has been characterized as an estrogen receptor $\beta$ agonist, and has been demonstrated in a phase 2 trial to be safe and more effective than a placebo in reducing the frequency and severity of VMS in postmenopausal women. The compound is entering phase 3 trials [44].

\section{Conclusions}

Menopause-related VMS are very common and can be associated with a high burden on patients and society. The physiology underlying VMS is complex and not fully understood, but it is clear that alterations in noradrenergic and serotonergic mechanisms during hypothalamic thermoregulation are involved in their development. Current treatments for VMS include hormonal therapy, TSECs, prescription medications developed for other indications, new estrogen receptor $\beta$ agonists, and CAM treatments. Short-term hormonal therapy has been shown to be essentially safe for the management of VMS, but the publicity given the WHI has substantially decreased the use of these treatments.

Treatment options for menopause-related VMS remain a significant unmet need. Until the precise physiological mechanism for VMS is elucidated, it is unlikely that a totally safe and effective therapy will be developed. Among women who are eligible for the treatment of menopauserelated VMS, $80 \%$ do not seek treatment, receive inadequate counseling, or do not have access to local medical aid. The development of therapies that specifically target VMS may provide high efficacy and reduce the risk of serious and potentially costly adverse events, thus increasing the overall cost-effectiveness of therapy.

Acknowledgment Acknowledgement is made to use of material from my own paper: Utian WH: Psychosocial and socioeconomic burden of vasomotor symptoms in menopause: A comprehensive review. Health and Quality of Life Outcomes 2005, 3:47. doi:10.1186/ 1477-7525-3-47. The electronic version of this article is the complete one and can be found online at http://www.hqlo.com/content/3/1/47.

Disclosure Conflicts of Interest: W. Utian: Consultant to Hygeia, Bionovo, Cleveland Clinic Foundation Innovations Center, Pharmavite, Merck, Sharp \& Dohme, Novogyne, Bayer, Lupin, Teva, and Pfizer.

\section{References}

Recently published papers of interest have been highlighted as:

- Of Importance

•- Of major Importance

1. United States Census 2000. Census 2000 profile [www.census. gov] US Department of Commerce. Economics and Statistics Administration. US Census Bureau; 2000, 2-5. 
2. Avis NE, Crawford SL, McKinlay SM. Psychosocial, behavioral, and health factors related to menopause symptomatology. Womens Health. 1997;3:103-20.

3. Mohyi D, Tabassi K, Simon J. Differential diagnosis of hot flashes. Maturitas. 1997;27:203-14.

4. Gold EB, Sternfeld B, Kelsey JL, Brown C, Mouton C, Reame N, Salamone L, Stellato R. Relation of demographic and lifestyle factors to symptoms in a multi-racial/ethnic population of women 40-55 years of age. Am J Epidemiol. 2000;152:463-73.

5. Ho SC, Chan SG, Yip YB, Cheng A, Yi Q, Chan C. Menopausal symptoms and symptom clustering in Chinese women. Maturitas. 1999;33:219-27.

6. Whiteman MK, Staropoli CA, Benedict JC, Borgeest C, Flaws JA. Risk factors for hot flashes in midlife women. J Womens Health (Larchmt). 2003;12:459-72.

7. Whiteman MK, Staropoli CA, Langenberg PW, McCarter RJ, Kjerulff KH, Flaws JA. Smoking, body mass, and hot flashes in midlife women. Obstet Gynecol. 2003;101:264-72.

8. Staropoli CA, Flaws JA, Bush TL, Moulton AW. Predictors of menopausal hot flashes. J Womens Health. 1998;7:1149-55.

9. Nachtigall LE, Nachtigall MJ. Menopausal changes, quality of life, and hormone therapy. Clin Obstet Gynecol. 2004;47:485-8.

10. Treatment of menopause-associated vasomotor symptoms: position statement of The North American Menopause Society. Menopause 2004, 11:11-33.

11. Feldman BM, Voda A, Gronseth E. The prevalence of hot flash and associated variables among perimenopausal women. Res Nurs Health. 1985;8:261-8.

12. Deecher DC. Physiology of thermoregulatory dysfunction and current approaches to the treatment of vasomotor symptoms. Expert Opin Investig Drugs. 2005; 14:434-48.

13. Kronenberg F. Hot flashes: epidemiology and physiology. Ann N Y Acad Sci. 1990;592:52-86.

14. Freedman RR. Pathophysiology and treatment of menopausal hot flashes. Semin Reprod Med. 2005;23:117-25.

15. Utian WH, Janata JW, Kingsberg SA, Patrick LD: Determinants and quantification of quality of life after the menopause: the Utian Menopause Quality of Life score. In The Menopause at the Millenium. Edited by Aso T and Yanaihara T. Taylor \& Francis; 2000:141-144.

16. Vliet EL. Menopause and perimenopause: the role of ovarian hormones in common neuroendocrine syndromes in primary care. Prim Care. 2002;29:43-67. vi.

17. McVeigh C. Perimenopause: More than hot flashes and night sweats for some Australian women. J Obstet Gynecol Neonatal Nurs. 2005;34:21-7.

18. Utian WH, Janata JW, Kingsberg SA, Schluchter M, Hamilton JC. The Utian Quality of Life (UQOL) Scale: development and validation of an instrument to quantify quality of life through and beyond menopause. Menopause. 2002;9:402-10.

19. Bobula JD. Vasomotor symptoms and quality of life (QoL) in postmenopausal women. Value Health. 2003;6:707.

20. Polo-Kantola P, Erkkola R. Sleep and the menopause. J Br Menopause Soc. 2004;10:145-50.

21. Moe KE. Hot flashes and sleep in women. Sleep Med Rev. 2004;8:487-97.

22. Shepherd JE. Effects of estrogen on cognition mood, and degenerative brain diseases. J Am Pharm Assoc (Wash ). 2001;41:221-8.

23. Hersh AL, Stefanick ML, Stafford RS. National use of postmenopausal hormone therapy: annual trends and response to recent evidence. JAMA. 2004;291:47-53.

24. •- Utian WH. (Panel Chair) Estrogen and progestogen use in postmenopausal women: 2010 position statement of The North American Menopause Society. Menopause 2010;17:242-255. The NAMS position papers on hormone therapy are considered the most internationally authoritative, and the most regularly updated, summaries of the current state of knowledge.
25. •• Santen RJ, Utian WH et al: Postmenopausal Hormone Therapy: An Endocrine Society Scientific Statement. J. Clin. Endocrinol. Metab. 2010;95:S1-S66. For the NAMS 2010 Hormone Therapy Position Paper, NAMS partnered with the Endocrine Society. The latter invited key background papers, published in this referenced supplement, which were then utilized by the NAMS HT Expert Panel in the development of their position paper.

26. Komm BS. A new approach to menopausal therapy: the tissue selective estrogen complex. Reprod Sci. 2008;15(10):984-92.

27. Bolognese M, Krege JH, Utian WH, Feldman R, Broy S, Meats DL, Alam M, Lakshmanan M, Omizo M. Effects of arzoxifene on bone mineral density and endometrium in postmenopausal women with normal or low bone mass. J Clin Endocrinol Metab. 2009;94:2284-9.

28. Kharode Y, Bodine PV, Miller CP, Lyttle CR, Komm BS. The pairing of a selective estrogen receptor modulator, bazedoxifene, with conjugated estrogens as a new paradigm for the treatment of menopausal symptoms and osteoporosis prevention. Endocrinology. 2008;149(12):6084-91.

29. •• Pinkerton JV, Utian WH, Constantine GD, Olivier S, and Pickar $\mathrm{JH}$. Relief of vasomotor symptoms with the tissue-selective estrogen complex bazedoxifene/conjugated estrogens: a randomized controlled trial. Menopause 2009;16:1116-1124. The first combination of a SERM with an estrogen has resulted in a synergistic combination having most of the benefits of hormonal therapy but potentially fewer of the risks. This reference and references [30••] and [31.・] are the key basic investigations demonstrating the safety and efficacy of the combination.

30. •• Pinkerton JV, Archer DF, Utian WH, Menegoci JC, Levine AB, Chines AA, and Constantine GD. Bazedoxifene effects on the reproductive tract in postmenopausal women at risk for osteoporosis. Menopause 2009;16:1102-1108. The first combination of a SERM with an estrogen has resulted in a synergistic combination having most of the benefits of hormonal therapy but potentially fewer of the risks. This reference and references [29・•] and [31・•] are the key basic investigations demonstrating the safety and efficacy of the combination.

31. • Archer DF, Pinkerton JV, Utian WH, Menegoci JC, de Villiers TJ, Yuen CK, Levine AB, Chines AC, and Constantine GD. Bazedoxifene, a selective estrogen receptor modulator: effects on the endometrium, ovaries, and breast from a randomized controlled trial in osteoporotic postmenopausal women. Menopause 2009;16:1109-1115. The first combination of a SERM with an estrogen has resulted in a synergistic combination having most of the benefits of hormonal therapy but potentially fewer of the

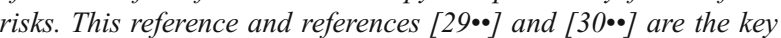
basic investigations demonstrating the safety and efficacy of the combination.

32. Fugate SE, Church CO. Nonestrogen treatment modalities for vasomotor symptoms associated with menopause. Ann Pharmacother. 2004;38:1482-99.

33. Kimmick GG, Lovato J, McQuellon R, Robinson E, Muss HB. Randomized, double blinded, placebo controlled, cross-over study of sertraline (Zoloft) for the treatment of hot flashes in women with early stage breast cancer taking tamoxifen. Breast J. 2006;12:114-22.

34. Loprinzi CL, Sloan JA, Perez EA, et al. Phase III evaluation of fluoxetine for treatment of hot flashes. J Clin Oncol. 2002;20:157883.

35. Nelson HD, Vesco KK, Haney E, et al. Nonhormonal therapies for menopausal hot flashes; systemic review and meta-analysis. JAMA. 2006;295:2057-71.

36. Stearns V, Beebe KL, Iyengar M, Dube E. Paroxetine controlled release in the treatment of menopausal hot flashes: a randomized controlled trial. JAMA. 2003;289:2827-34.

37. • Archer D, Dupont CM, Constantine GD, Pickar JH, Olivier S; Study 319 Investigators. Desvenlafaxine for the treatment of 
vasomotor symptoms associated with menopause: a double-blind, randomized, placebo-controlled trial of efficacy and safety. Am J Obstet Gynecol. 2009;200(3):238.

38. Joffe H, Petrillo L, Viguera A, Koukopoulos A, Silver-Heilman K, Farrell A, Yu G, Silver M, Cohen LS. Eszopiclone improves insomnia and depressive and anxious symptoms in perimenopausal and postmenopausal women with hot flashes: a randomized, double-blinded, placebo-controlled crossover trial. Am J Obstet Gynecol. 2010;202(2):171.e1-171.e11.

39. Butt DA, Lock M, Lewis JE, Ross S, Moineddin R. Gabapentin for the treatment of menopausal hot flashes: a randomized controlled trial. Menopause. 2008;15(2):310-8.

40. Kronenberg F, Fugh-Berman A. Complementary and alternative medicine for menopausal symptoms: a review of randomized, controlled trials. Ann Intern Med. 2002;137:805-13.

41. •- Clarkson TB, Utian WH, Barnes S, et al. for the NAMS Isoflavone Translational Symposium Panel. The role of soy isoflavones in menopausal health: report of The North American Menopause Society/Wulf H. Utian Translational Science Symposium in Chicago, IL. (October, 2010). Menopause 2011; 18: 732753. This reference is the most current summary of the entire background science regarding the use of soy isoflavonoids for postmenopausal women.

42. Levis S, Strickman-Stein N, Ganjei-Azar P, Xu P, Doerge DR, Krischer J. Soy isoflavones in the prevention of menopausal bone loss and menopausal symptoms: a randomized, double-blind trial. Arch Intern Med. 2011;171(15):1363-9.

43. Stovall DW, Pinkerton JP. MF-101, an estrogen receptor $\beta$ agonist for the treatment of vasomotor symptoms in peri- and postmenopausal women. Curr Opin Inv Drugs. 2009;10:365-71.

44. Grady D, Sawaya GF, Johnson KC, Koltun W, Hess R, et al. MF 101 , a selective estrogen receptor $\beta$ modulator for the treatment of menopausal hot flashes: a phase II trial. Menopause. 2009;16:45865. 